\title{
Study on Supplier Selection Based on the Collaborative Mechanism and Game Strategy ${ }^{*}$
}

\author{
Zhongmin Fang ${ }^{1}$, Zhiya Chen ${ }^{1}$, Minghua Zeng ${ }^{2}$ \\ ${ }^{1}$ School of Traffic \& Transportation, Central South University, Changsha, China \\ ${ }^{2}$ East China Jiaotong University, Nanchang, China \\ Email: Fzm1968@163.com
}

Received August 7, 2012; revised September 7, 2012; accepted September 15, 2012

\begin{abstract}
Based on the fluctuation of production efficiency, evaluating synergetic model of the supplier collaboration degree has been set up, and sequence participation amount of the model is analyzed. Combined with the game strategy and collaborative mechanism of supply chain, supply chain game strategy model is built to make the allocation of the market resources more effective when production efficiency is consistent, and the model of supplier selection and the algorithm of solving the model are put forward, investigating the game process of supplier selection from the profit and social welfare of the supplier and the demand. In the end, the efficiency of the model and algorithm is validated by a case simulation.
\end{abstract}

Keywords: Supplier Selection; Collaborative Mechanism; Game Strategy; Social Welfare; Genetic Algorithms

\section{Introduction}

With the increasingly high degree of productive specialization, the companies are increasingly focusing on their core business, so a lot of parts are processed from external sourcing or outsourcing. This manufacturing model calls for enterprises to cooperate with a growing number of suppliers, so that the problem of evaluation and selection of suppliers is constantly given new meaning and becomes an important research topic, and gets more and more attention in academia and the business community [1-3].

In consistent with production efficiency, without considering senseless loss, by determining the price level we can increase the sum of suppliers' production surplus and consumers' demand surplus, namely, social welfare. So that it makes the market allocation of resources more effective, and so it is very necessary that we inspect the synergy and game process of supplier selection from the profits and social welfare of the supplier and the demander.

\section{The Model of Supplier Coordination Me- chanism}

Suppliers' production efficiency has an effect on the demanders' production efficiency and the supplier's choice

\footnotetext{
*The work is supported by HNPSSF Grant\#2010YBB246 to Fang Zhongmin and HNNSF Grant \#09JJ3135 to Fang Xiaoping and JXDESF Grant \# GJJ12322 to Zeng Minghua.
}

strategy [4], at the same time, the competition pressure of the external suppliers affects the supplier's production efficiency, shown in two aspects: 1) The competition and synergy of different suppliers' production efficiency not only promotes the suppliers to enhance the efficiency of production on a certain basis, but also makes them be in a competitive position on the choice of the demander enterprise; 2) The choice of suppliers competition strategy, that is using a different price system, decides the production batch.

Demander enterprise's demand of a product is affected by overall production efficiency of the supplier system [5], that is, the influence of social average production time, without considering the effect of alternative products; Because the social average production efficiency is the function of the supplier's production efficiency [6], therefore, we can draw a conclusion that the production efficiency of the demander will be affected by the supplier.

\subsection{Synergy Mechanism of Uncertainty of Production Efficiency}

Here, we suppose the $Q_{i}$ as the $i$ th supplier's production batch; $l_{t}$ as the lead time of the demander to supplier and it embodies the characteristic of flexible time; $q_{i}$ as the ith suppliers' production efficiency; $\varepsilon_{i}^{q}$ as the value of random production efficiency caused by other factors for the suppliers, and obeys the normal distribution $N\left(\mu_{i}^{q}, \sigma_{i}^{q}\right) ; E_{i}^{q}$ as the influence functions of 
the supplier and demander, suppliers about other production efficiency and growth rates; $D_{t+l t}$ as the demand of the demander in the lead time; $\varepsilon^{d}$ as the random demand of the demander caused by other factors, and it obeys the normal distribution $N\left(u^{D}, \sigma^{D}\right) ; E^{D}$ as the demander's influence functions caused by supplier production efficiency.

According to the theory of Haken Synergy Mechanism [7-10], we can build the uncertain model of production efficiency, as Formular (1):

$$
\left\{\begin{array}{l}
q_{i}=\frac{\partial Q_{i}}{\partial t}+\varepsilon_{i}^{q}+E_{i}^{q}\left(d, q_{1}, \cdots, q_{i-1}, q_{i+1}, q_{n}\right) \\
d=\frac{\partial D(l t)}{\partial t}+\varepsilon^{d}+E^{D}\left(q_{1}, \cdots, q_{n}\right)
\end{array}\right.
$$

If we consider about the influence of time flexible, and the equation of lead time function, we can generalize as Formular (2):

$$
\chi_{t}=\frac{\partial l t}{\partial t}+\varepsilon^{l t}+E\left(d, q_{i}\right)
$$

And so the function based on the uncertainty of production efficiency, we can generalize as Formular (3):

$$
\left\{\begin{array}{l}
q_{i}=\frac{\partial Q_{i}}{\partial t}+\varepsilon_{i}^{q}+E_{i}^{q}\left(d, q_{1}, \cdots, q_{i-1}, q_{i+1}, q_{n}, l t\right) \\
d=\frac{\partial D(l t)}{\partial t}+\varepsilon^{d}+E^{D}\left(q_{1}, \cdots, q_{n}, l t\right) \\
\chi_{t}=\frac{\partial l t}{\partial t}+\varepsilon^{l t}+E\left(d, q_{i}\right)
\end{array}\right.
$$

When using the demand pull type to produce, the lead time namely the time flexibility is the order parameter, according to the corresponding elimination method we can solve the equations; when using the push type to produce, we need to inspect the location in which supplier and enterprise can be in the supply chain, if the supplier has a larger advantage, the variable $q_{i}$ is the order parameter; if the enterprise has the larger advantage, the variable $d$ is the order parameter; similarly, we can use the adiabatic elimination method to solve equations.

\subsection{The Evaluation Model of Supplier Operation Synergy}

The degree of supplier operation synergy is the embodiment of the synergy degree between the largest supplier in the lead time and demander enterprise, available equation as follows:

$$
\alpha=\max \left(\frac{\min \left(\max \left(Q_{i}\right), D\right)}{\max \left(\max \left(Q_{i}\right), D\right)}\right)
$$

\section{Game Strategy Model of Supply Chain}

When it comes to production efficiency, the supplier's competition can promote the supplier to increase their ability, and enhance the social production efficiency, reflect the change process between the competition for a long time and synergy of suppliers, but failed to reflect the game process between the supplier and the demander in the short term. When it comes to select suppliers, there is some game strategy in suppliers whose production efficiency is more uniform, and suppliers use different game strategy to get the order, so as to provide the product demand and production service to the demand. Here we assume the relationship between the supplier and the demander enterprises as the complete information extension game [11], so it is feasible to use the method of game theory to study supplier competition strategy model in the supplier selection process.

When production efficiency is consistent, according to the principle of microeconomics [12], with the supply and demand determined, without considering senseless loss, we can increase the sum of suppliers' production surplus and demander's consumer surplus, namely, social welfare, by determining the price level. So that it makes the market allocation of resources more effective, and so we consider the game process of supplier selection from the profits and social welfare of the supplier and the demander.

\subsection{Game Strategy Model of Supply Chain}

We assume $p_{i}^{s}$ as unit cost of supplier's raw materials; $c_{i}^{s}$ as manufacturing and management apportioned cost, the function about production batch, and the production batch is bigger then the manufacturing and management apportioned cost is smaller, with the increase of production batch, enterprise load increases, and manufacturing and management apportioned cost of enterprise increases; $c$ as the enterprise manufacturing and management apportioned cost, by the same reason is function about production batch; $p$ as unit material purchase cost; $p^{d}$ as the price of unit product of the demand; $Q$ as the demand of market, and it is a function of time; $e$ as the raw materials demand of unit product; $B$ as the profits of the demand; $B_{i}^{s}$ as the profit of supplier; $W$ as social welfare.

In the process of supply chain game, there is the game process as follows, and its target value process shown in Table 1.

Because of the different production efficiency of supplier, the additional cost will change with the change of production efficiency, so we add the additional cost $c_{i}^{s}\left(Q_{i} / t\right)$ to provoke the supplier to accelerate production speed, to increase the efficiency. So we have: 
Table 1. Function value of welfare during game process.

\begin{tabular}{|c|c|c|c|}
\hline & \multicolumn{3}{|c|}{ Demand Enterprise } \\
\hline \multirow{3}{*}{ Supplier } & & Not Betray & Betray \\
\hline & Not Betray & overall welfare function & Welfare function demand enterprise lead \\
\hline & Betray & Welfare function supplier lead & Welfare is 0 and cannot reach any deal \\
\hline
\end{tabular}

$$
\left\{\begin{array}{l}
B=\left[P^{d}-p-c(Q)\right] \cdot Q \\
B_{i}^{s}=\left[p-p_{i}^{s}-c_{i}^{s}\left(Q_{i} / t\right)\right] \cdot Q_{i}
\end{array}\right.
$$

1) If we consider the welfare from the whole supply chain, we can get:

$$
W=P^{d} \cdot Q-\left(p_{i}^{s}+c_{i}^{s}\left(\mathrm{e} \cdot Q_{i} / t\right)\right) \cdot \mathrm{e} \cdot Q
$$

If we want to get the largest supplier total production surplus, we transform into solving the binary implicit function extreme problems about $B_{i}^{s}$ to $Q, p_{i}$. At this time $p_{i}$ is a setting value $p_{0}$, so it can be converted into the condition extremum problems:

$$
\left\{\begin{array}{l}
f\left(p_{i}, Q\right)=\sum_{i} B_{i}^{s} \\
\varphi\left(p_{i}, Q\right)=p_{i}-p_{0}=0
\end{array}\right.
$$

And then, there is only $p_{i} \leq p_{0}$ for supplier, and it can form the supplier producer surplus.

If we can obtain the maximum value of $B_{i}^{s}$ at the stagnation point $\left(p_{0}, Q_{1}\right)$, and the consumer surplus of the demand is $B\left(p_{0}, Q_{1}\right)$, and so the overall welfare is $B\left(p_{0}, Q_{1}\right)+B_{i}^{s}\left(p_{0}, Q_{1}\right)$.

2) If we want the consumer surplus to be the largest one, so we transform into solving the binary implicit function extreme problems about $B$ to $Q, p_{i}$ :

$$
\left\{\begin{array}{l}
f(p, Q)=B \\
\varphi(p, Q)=p-p_{0}^{\prime}=0
\end{array}\right.
$$

And then, there is only $p_{i} \leq p_{0}$ for supplier, and it can form the demander enterprise consumer surplus.

If we can obtain the maximum value of $B$ at the stagnation point $\left(p_{0}^{\prime}, Q_{2}\right)$, and the consumer surplus of demander enterprise is $B_{i}^{s}\left(p_{0}^{\prime}, Q_{2}\right)$, and so the overall welfare is $B\left(p_{0}^{\prime}, Q_{2}\right)+B_{i}^{s}\left(p_{0}, Q_{2}\right)$.

3 ) If we want the welfare to be the largest one, so we transform into solving the binary implicit function extreme problems about $W$ to $Q$ :

$$
\left\{\begin{array}{l}
f(W, Q)=W \\
\varphi(W, Q)=W-P^{d} \cdot Q+\left(p_{i}^{s}+c_{i}^{s}\left(\mathrm{e} \cdot Q_{i} / t\right)\right) \cdot \mathrm{e} \cdot Q=0
\end{array}\right.
$$

And we can get the largest value $W_{0}$ of $W$ at $\left(p_{0}, Q_{0}\right)$.

In the supply chain, we should make sure the benefits maximized, so the game strategy model of supply chain defined by the sum of the benefits of supplier and de- mand enterprise is:

$$
\begin{aligned}
& B_{c}=\max ( B\left(p_{0}, Q_{1}\right)+B_{i}^{s}\left(p_{0}, Q_{1}\right), \\
&\left.B\left(p_{0}^{\prime}, Q_{2}\right)+B_{i}^{s}\left(p_{0}^{\prime}, Q_{2}\right), W_{0}\right)
\end{aligned}
$$

\subsection{Model Solution}

The game strategy model in the cooperation supply and demand enterprise, setting case (3) for example, the solving process is as follows:

$$
\left\{\begin{array}{l}
f(W, Q)=W \\
\varphi(W, Q)=W-P^{d} \cdot Q+\left(p_{i}^{s}+c_{i}^{s}(\mathrm{e} \cdot Q / t)\right) \cdot \mathrm{e} \cdot Q=0
\end{array}\right.
$$

1) Getting the derivative, stagnation and special points of function $\varphi(W, Q)$, namely, the point set $M^{\prime}$ meets the condition $\frac{\mathrm{d} Q}{\mathrm{~d} t}=0$

$$
\begin{aligned}
\varphi(W, Q) & =\frac{\mathrm{d} W}{\mathrm{~d} Q} \cdot \frac{\mathrm{d} Q}{\mathrm{~d} t}-P^{d} \cdot \frac{\mathrm{d} Q}{\mathrm{~d} t}+\left(p_{i}^{s}+c_{i}^{s}(\mathrm{e} \cdot Q)\right) \cdot \mathrm{e} \cdot \frac{\mathrm{d} Q}{\mathrm{~d} t} \\
& +\mathrm{e}^{2} \cdot Q \cdot \frac{\mathrm{d} c_{i}^{s}(Q)}{\mathrm{d} t}=0
\end{aligned}
$$

It can arrange to be:

$$
\begin{aligned}
\frac{\mathrm{d} W}{\mathrm{~d} Q}= & \left\{P^{d} \cdot \frac{\mathrm{d} Q}{\mathrm{~d} t}-\left(p_{i}^{s}+c_{i}^{s}(\mathrm{e} \cdot Q / t)\right) \cdot \mathrm{e} \cdot \frac{\mathrm{d} Q}{\mathrm{~d} t}\right. \\
& \left.-\mathrm{e}^{2} \cdot Q \cdot \frac{\mathrm{d} c_{i}^{s}(Q)}{\mathrm{d} t}\right\} / \frac{\mathrm{d} Q}{\mathrm{~d} t}
\end{aligned}
$$

Through the equation set:

$$
\left\{\begin{array}{l}
\varphi(W, Q)=0 \\
\varphi^{\prime}(W, Q)=0
\end{array}\right.
$$

2) Deciding the symbol of $\varphi^{\prime \prime}(W, Q)$, combined with the counter-evidence method to analyze the point's function value at $\frac{\mathrm{d} Q}{\mathrm{~d} t}=0$, we can get the extreme value using the second sufficient conditions of extremum existence.

\section{Supplier Selection Method}

The method of supplier selection is on the basis of the ability of supplier operation synergy and the social welfare values of supplier chain. So selecting suppliers, we can set up the multi-objective programming model of 
supplier selection. Because the value range of the supplier operation synergy model is $0<\alpha \leq 1$, so we use multiplier method to solve the multi-objective programming model effectively. Because the suppliers' levels are different, here we ask the operation synergy ability $a$ must be larger than the minimum standard $a_{0}$, and eliminate the supplier directly who do not meet the requirements, and so we do not need to get the value in supplier selection multi-objective programming model.

1) Supplier selection multi-objective programming model

To sum up, supplier selection multi-objective programming model is:

$$
Z=\max \left(\alpha \cdot B_{c}\right)
$$

2) Solvement of Model

Step 1: data initialization;

Step 2: solving the supplier operation synergy ability, and classifying the supplier by range $(0,0.1]$,

$(0.1,0.2], \cdots,(0.9,1]$ into ten ranges, and eliminating the supplier whose ability is lower than $a_{0}$, and storing the classification supplier whose ability is upper than $a_{0}$;

Step 3: solving the social welfare of supply chain;

Step 4: according to the formula $Z=\max \left(\alpha \cdot B_{c}\right)$ to get the optimum relation, that is qualified supplier;

Step 5: outputting the qualified supplier, and then the end.

\section{Sample Analysis}

During a mechanical equipment manufacturing process, in the supply chain, there is a manufacturer, and there are 10 suppliers for choice. We assume the supplier extra product cost function is $r \cdot\left(l t_{i}+t\right) / Q$, the A product manufacturers need 10 days to finish 100 units of the product $A$, and the supplier's unit price is 20,000 . To manufacture product $A$, we need raw materials $B$ and its demand is 50. After order placement, the time that the supplier needed to prepare materials is the vector hours It , It $=\{100,400,200,95,81,77,120,130,111,115\}$, and the cost price of suppliers is

$p_{i}^{s}=(20,16,35,15,16,24,25,26,27,22)$, and we set the deal unit price as $p_{0}=30$, and the increasing value of each piece of raw materials in product $\mathrm{A}$ is $\Delta V=60$. Without considering the influence of random factors and lead time, we establish the following equation:

$$
\left\{\begin{array}{l}
q_{i}=50 / t+E_{i}^{q}\left(d, q_{1}, \cdots, q_{i-1}, q_{i+1}, q_{n}\right) \\
d=100 /\left(t-l t_{i}\right)+E^{D}\left(q_{1}, \cdots q_{n}\right)
\end{array}\right.
$$

And then, we assume the manufacturer as the lead enterprise, so the order parameter is $d$.

As shown in Figure 1, we use the tool to draw the contrast of the production efficiency (the first supplier and manufacturer).
When manufacturers do not select the qualified supplier, the supplier produces the corresponding products according to the market demand, and the market demand always changes as the change of time. So according to game strategy model, we can give equations:

$$
\left\{\begin{array}{l}
f\left(p, Q_{i}\right)=\left[p-p_{i}^{s}-r \cdot\left(l t_{i}+t\right) / 50\right] \cdot Q_{i} \\
\varphi\left(p, Q_{i}\right)=p-30=0
\end{array}\right.
$$

We can get it that the first equation of the equation set is the implicit function about time. So we can get the changing relationship of the supplier's production surplus and the time. We can get the relationship of social welfare and production time, shown in the following Figure 2.

Finally, we choose the fifth supplier as the qualified one after calculation, and the social welfare is 4580 , and the synergy degree is 0.95, as shown in Figure 3.

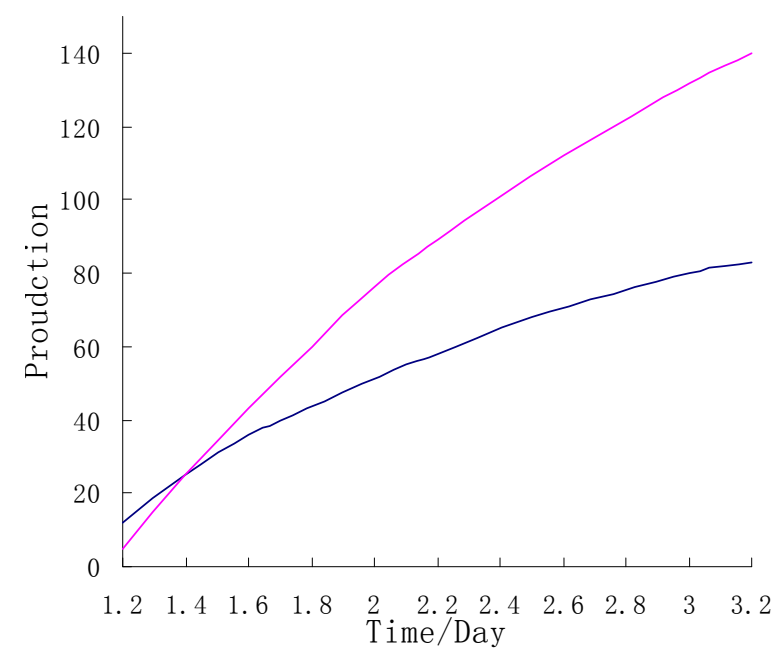

Figure 1. Synergy evolution process of production efficiency of the first supplier and manufacturer.

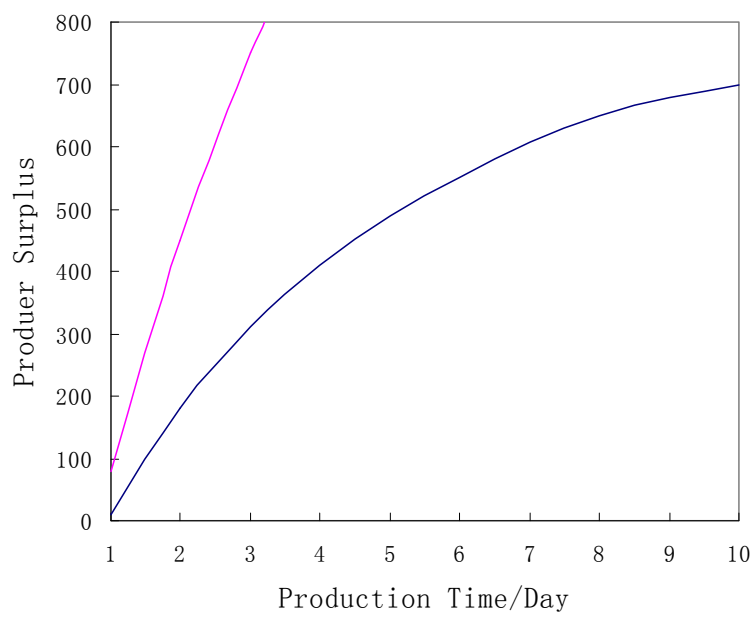

Figure 2. The changing relationship of production surplus and time while selecting different suppliers. 

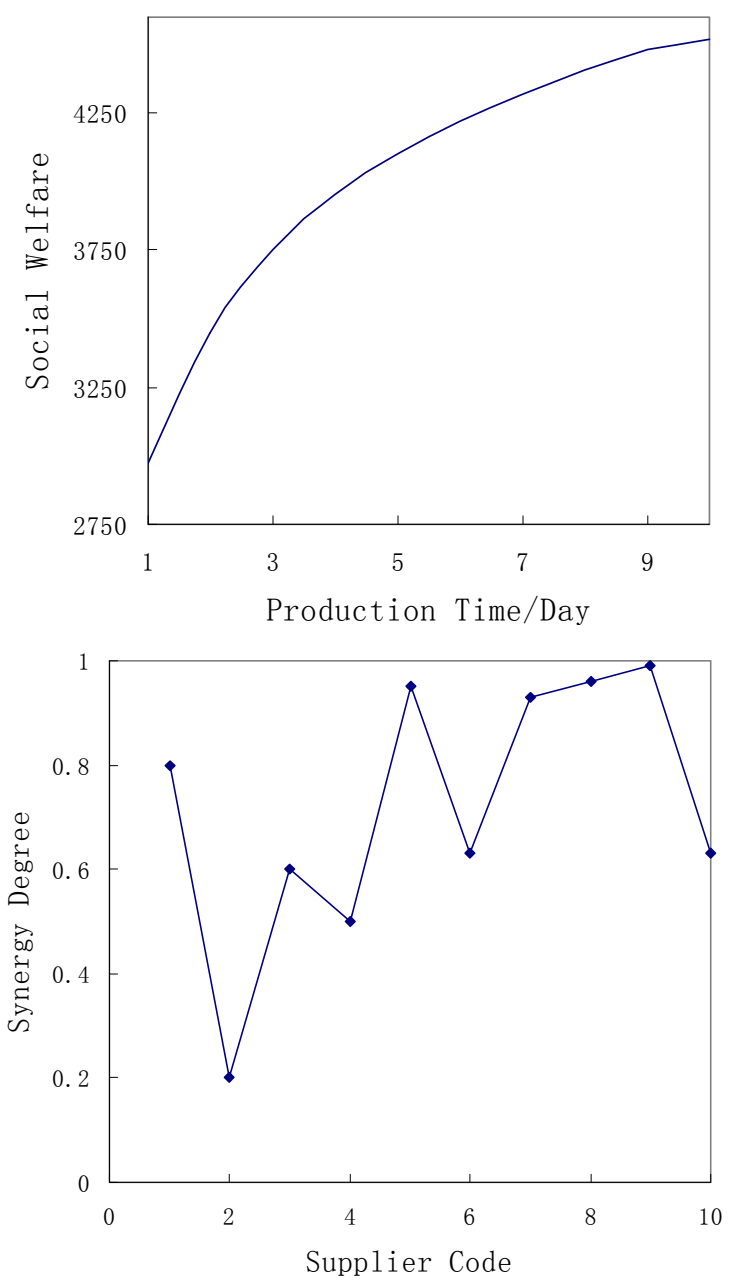

Figure 3. The analysis of supplier selection.

\section{Conclusion}

This paper analyzes the supplier synergy mechanism, and due to the volatility of the production efficiency, and builds the evaluation synergy model of the supplier operation synergy degree, and analyzes the order parameter of model. In this paper, we combined with the game strategy and synergy mechanism of supply chain, in consistent with production efficiency, and to make market allocation of resources more effective, then build the game model of supply chain, and inspect the game proc- ess of the supplier selection from the perspective of the profits and social welfare of the supplier and the demand business; Finally we put forward the supplier selection model and the algorithm of model solvement.

\section{REFERENCES}

[1] J.-X. Jia, "Research on Production Ability and Pricing Strategy in Supply Chain Network, "Computer Integrated Manufacturing System, Vol. 14, No. 10, 2008, pp. 20362045.

[2] L. Qian, “An ANN Pruning Algorithm Based Approach to Vendor Selection," Journal of Systems Management (USA), Vol. 46, No. 5, 1995, pp. 314-320.

[3] H.-L. Guo, Y.-X. Hou and B.-H. Yang, “Competitive \& Cooperation Game-Theory Coordination Model of ThreeHierarchy Supply Chain with M Suppliers, 1 Manufacturer and N Retailors," Chinese Journal of Management Science, Vol. 16, No. 6, 2008, pp. 54-60.

[4] Q.-W. Jiang, "Research on Optimal Method for Supplier Selection under Uncertain Environment,” Ph.D. Thesis, Central South University, Changsha, 2011.

[5] J.-J. Zhu, S.-X. Liu, M.-G. Wang and M. Huang, “Analyses of Vendor Selection and Order Planning," Journal of Northeastern University, Vol. 24, No. 10, 2003, pp. 956958.

[6] X. Liu, H.-Y. Li, C.-E. Wang and C.-B. Chu, "A Survey of Supplier Selection Model and Approaches," Chinese Journal of Management Science, Vol. 12, No. 1, 2004, pp. 139-148.

[7] H. Haken, “Advanced Synergetics,” Springer-Verlag, Berlin, 1987.

[8] H. Haken, "Synergetics: An Introduction," 3th Edition, Springer, Berlin, 1983.

[9] H. Haken, "Information and Self-Organization: A Macroscopic Approach to Complex Systems," Springer, Berlin, 2006.

[10] H. Haken, "Cooperative Phenomena in System Far from Thermal Equilibrium and in Nonphysical Systems,” Reviews of Modern Physics, Vol. 47, No. 1, 1975, pp. 67121. doi:10.1103/RevModPhys.47.67

[11] M. J. Osborn and A. Robinstein. "A Course in Game Theory,” China Social Science Press, Beijing, 2000.

[12] N. G. Mankiw, "Principles of Economics," 5th Edition, Peking University Press, Beijing, 2009. 